\title{
Characterizing gamma fields using isomeric activation ratios
}

\author{
Ramkumar Venkataraman *, Ronald F. Fleming \\ Department of Nuclear Engineering, The Universtty of Michtgan, Ann Arbor, MI, USA
}

\begin{abstract}
Isomeric activities were induced in indium by gamma irradiation in three different gamma fields, through the reactions ${ }^{115} \operatorname{In}\left(\gamma, \gamma^{\prime}\right)^{115 \mathrm{~m}}$ In and ${ }^{113} \ln \left(\gamma, \gamma^{\prime}\right)^{113 \mathrm{~m}} \mathrm{In}$. The irradiation fields were (i) the $15 \mathrm{kCi}{ }^{60} \mathrm{Co}$ source available in the University, (ii) the spent fuel gamma irradiator in the pool of the University's Ford Nuclear Reactor (FNR) and (iii) south face of the core of the FNR during routine shut downs. Isomeric activation ratios can serve to characterize gamma fields, provided the response functions of the two $\left(\gamma, \gamma^{\prime}\right)$ reactions sample different energy regimes of the gamma spectrum present in the irradiation fields. The response of an isomeric activation detector, in turn, depends on the number of activation energy levels of the nuclide and the probabilities with which the activation levels de-populate to the isomeric level. The reaction rate ratio $R_{\text {In } 115 \mathrm{~m}} / R_{\text {In } 113 \mathrm{~m}}$ was measured in the three gamma fields. The measured ratios were (i) $1.210 \pm 0.011$ in the ${ }^{60} \mathrm{Co}$ source, (ii) $1.314 \pm 0.060$ in the spent fuel gamma irradiator and (iii) $1.298 \pm 0.039$ in a location alongside the FNR core during routine shut downs. The measured reaction rate ratios are not only close to each other, but close to unity as well. This indicates that the excitation functions for the reactions ${ }^{115} \operatorname{In}\left(\gamma, \gamma^{\prime}\right)^{115 \mathrm{~m}} \operatorname{In}$ and ${ }^{113} \operatorname{In}\left(\gamma, \gamma^{\prime}\right){ }^{113 \mathrm{~m}} \operatorname{In}$ have similar shapes and that for the nuclides ${ }^{115} \mathrm{In}$ and ${ }^{113} \mathrm{In}$, the number of activation energy levels and the probabilities with which they populate the isomeric levels are very similar to each other. Thus, the ratio $R_{\operatorname{In} 115 \mathrm{~m}} / R_{\operatorname{In} 113 \mathrm{~m}}$ will not yield any information regarding the shape of gamma spectrum in the field of measurement. However by choosing $\left(\gamma, \gamma^{\prime}\right)$ reactions with different shapes for the excitation functions one can measure a set of isomeric activation ratios that characterize a given gamma field.
\end{abstract}

\section{Introduction}

It is well known that isomeric activities can be induced in many stable nuclides that occur in the region of closed nuclear shells, by gamma activation [1-8]. The $\left(\gamma, \gamma^{\prime}\right)$ reaction excites the target nucleus from the ground state to one of its activation energy levels above the metastable isomeric state. This will occur only if the gamma spectrum in the irradiation field has photons whose energies matched one or more activation energy levels of the target nucleus. Once in the excited state, the nucleus can then de-excite to populate the isomeric state, with a transition probablity that is dictated by the spin and parity of the nuclear states. From the metastable state, the nucleus decays to its ground state by emitting gammas or conversion electrons. The response of a $\left(\gamma, \gamma^{\prime}\right)$ reaction depends on the number of activation energy levels that are available. The reaction rate per atom from a $\left(\gamma, \gamma^{\prime}\right)$ reaction is given by

$R=\left\langle\sigma_{\gamma}\right\rangle \Phi$

\footnotetext{
* Corresponding author.
}

where $\left\langle\sigma_{\gamma}\right\rangle$ is the integrated cross-section averaged over the gamma spectrum and $\Phi$ is the total gamma flux:

$\left\langle\sigma_{\gamma}\right\rangle=\frac{\int_{0}^{\infty} \sigma\left(E_{\gamma}\right) \phi\left(E_{\gamma}\right) \mathrm{d} E_{\gamma}}{\int_{0}^{\infty} \phi\left(E_{\gamma}\right) \mathrm{d} E_{\gamma}}$,

$\Phi=\int_{0}^{\infty} \phi\left(E_{\gamma}\right) \mathrm{d} E_{\gamma}$.

If two $\left(\gamma, \gamma^{\prime}\right)$ reactions are induced by irradiating the target materials simultaneously in a gamma field, the ratio of reaction rates per atom will be

$R_{1} / R_{2}=\left\langle\sigma_{\gamma}\right\rangle_{1} /\left\langle\sigma_{\gamma}\right\rangle_{2}$.

The ratio depends on the shapes of the cross-sections of the two reactions and the shape of the gamma spectrum in the irradiation field. However, it is independent of the magnitude of the gamma flux in the field of irradiation. If the cross-section shapes for the two $\left(\gamma, \gamma^{\prime}\right)$ reactions are different, the ratio $R_{1} / R_{2}$ will be sensitive to the spectral shape of the gammas. Experimentally, the reaction rate per atom can be determined from,

$R=A_{0}(1+\alpha) /\left(\epsilon \frac{m a N_{\mathrm{A}}}{A}\right)$, 
where $\alpha$ is the internal conversion coefficient, $\epsilon$ is the counting efficiency, $m$ is the elemental mass of the foil, $a$ is the isotopic abundance, $N_{\mathrm{A}}$ is the Avogadro's number, and $A$ is the atomic weight of the target element. The quantity $A_{0}$ is defined as,

$A_{0}=\frac{\lambda C \mathrm{e}^{\lambda t_{\mathrm{w}}}}{\left(1-\mathrm{e}^{-\lambda \tau}\right)\left(1-\mathrm{e}^{-\lambda\lrcorner}\right)}$,

where $C$ is the net counts in the gamma peak, $\lambda$ is the decay constant, $\tau$ is the irradiation time, $t_{\mathrm{w}}$ is the time elasped between the end of irradiation and the beginning of counting, and, $\Delta$ is the live counting time.

The ratio of the reaction rates per atom will then be,

$$
\frac{R_{1}}{R_{2}}=\frac{\left(\frac{A_{0}}{m}\right)_{1}}{\left(\frac{A_{0}}{m}\right)_{2}}\left(\frac{\epsilon_{2}}{\epsilon_{1}}\right)\left(\frac{1+\alpha_{1}}{1+\alpha_{2}}\right)\left(\frac{a_{2}}{a_{1}}\right)\left(\frac{A_{1}}{A_{2}}\right) .
$$

Thus, in principle, it is possible to measure a set of $(\gamma$, $\gamma^{\prime}$ ) reaction rate ratios that are unique to a specific gamma field. Integral cross-sections of the $\left(\gamma, \gamma^{\prime}\right)$ reactions are often determined by estimating or calculating the photon flux in the field of irradiation. The reaction rate ratio measurements can be used to verify such procedures.

\section{Experiment}

Isomeric activities were induced in the nuclides ${ }^{115} \mathrm{In}$ and ${ }^{113}$ In by gamma activation of indium ingots in three different gamma fields. The indium targets were cylindrical, with a diameter of $1.778 \mathrm{~cm}$ and a thickness of 0.635 $\mathrm{cm}$. A short description of the three irradiation fields is given below.

\section{1. ${ }^{60}$ Co source}

The ${ }^{60} \mathrm{Co}$ source at the Phoenix Memorial Laboratory in the University of Michigan is contained in a $16.5 \mathrm{ft}$ deep pool of water, at a depth of $11.5 \mathrm{ft}$. The source is made up of ten aluminum tubes packed with pellets of cobalt. The rods are $33 \mathrm{~cm}$ in length and are located on a circle of diameter $12.5 \mathrm{~cm}$. The center well is located on the top cover, at the same axis as the source. The center well is cylindrical, with an inner diameter of $8 \mathrm{~cm}$ and a height of $30 \mathrm{~cm}$. The indium ingots were irradiated in the center well at a distance of $7.6 \mathrm{~cm}$ from its bottom and $8.24 \mathrm{~cm}$ above the water surface. The source strength is $15 \mathrm{kCi}$ approximately. Fig. 1 is a schematic diagram of the source.

\subsection{Spent fuel gamma irradiator}

The spent fuel gamma irradiator is located at the west end of the reactor pool, $8 \mathrm{ft}$ away from the core and at a height of $10 \mathrm{ft}$ above the core. The facility consists of two

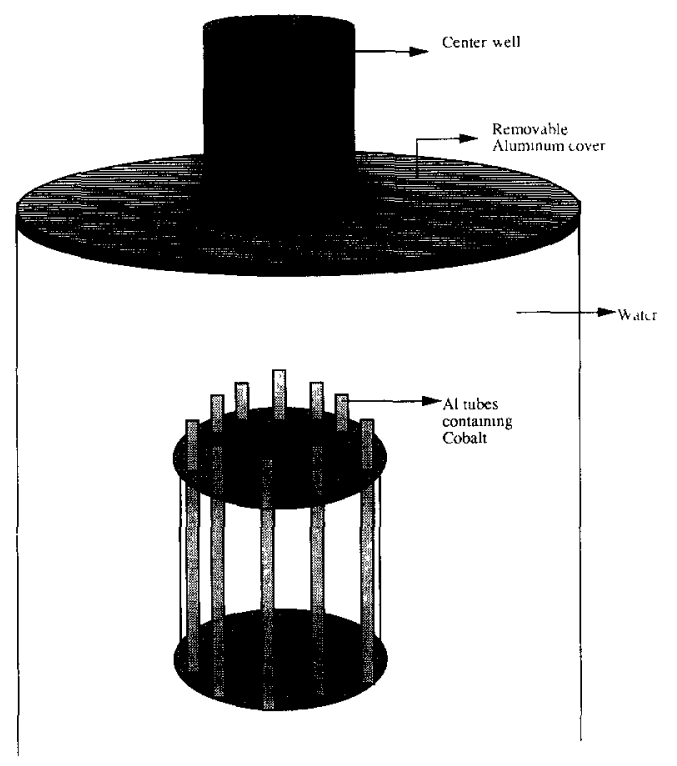

Fig. 1. A schematic diagram of the ${ }^{60} \mathrm{Co}$ source at Phoenix Memorial Laboratory, University of Michigan.

rows of six fuel elements each, with 6 in. of water between the rows. The indium ingot was centered in the irradiation facility, in all three directions.

\subsection{South-face of the reactor core}

Indium irradiations alongside the south-face of the reactor core were conducted inside the Materials Dosimetry Reference Facility (MDRF) [9] during routine shut downs. The isomeric activities of interest were induced by gammas from the decay of fission products in the fuel elements. The MDRF is actually a reference neutron field, intended for validating neutron dosimetry irradiations. It is a steel cylinder of $15 \mathrm{~cm}$ outer diameter and $5 \mathrm{~cm}$ inner diameter, surrounded by cadmium. An aluminum thimble was used to locate the indium ingot at the center of the facility. It was necessary to use this facility for gamma irradiations during shut downs, as it reduced the interference from the thermal neutron induced reaction ${ }^{115} \operatorname{In}(\mathrm{n}$, $\gamma)^{116 m} \mathrm{In}$. To monitor fast neutron fluence rates during these irradiations, nickel foils were irradiated along with indium and the activity from the ${ }^{58} \mathrm{Ni}(\mathrm{n}, \mathrm{p})^{58} \mathrm{Co}$ reaction was measured. The activities from the nickel foils revealed that the fast neutron fluence rates were equivalent to a reactor power of $2 \mathrm{~W}$ during shut downs.

\subsection{Counting techniques}

The isomeric activities from the reactions ${ }^{115} \operatorname{In}(\gamma$, $\left.\gamma^{\prime}\right)^{115 \mathrm{~m}}$ In and ${ }^{113} \operatorname{In}\left(\gamma, \gamma^{\prime}\right)^{113 \mathrm{~m}}$ In were measured by counting the $336 \mathrm{keV}$ and $392 \mathrm{keV}$ gammas that are emitted during the decay of the nuclear isomers ${ }^{115 \mathrm{~m}} \mathrm{In}$ and ${ }^{113 \mathrm{~m}} \mathrm{In}$, 
Table 1

Measured ratios of reaction rates per atom

\begin{tabular}{ll}
\hline Irradiation field & $R_{\operatorname{In} 115 \mathrm{~m}} / R_{\operatorname{In} 113 \mathrm{~m}}$ \\
\hline${ }^{60}$ Co Source & $1.210 \pm 0.011$ \\
Spent fuel gamma irradiator & $1.314 \pm 0.060$ \\
South face of reactor core & $1.298 \pm 0.039$ \\
$\quad$ during shut downs & \\
\hline
\end{tabular}

respectively. A high purity Ge detector system was employed to measure the count rates. Since the induced activities were low, the indium ingots were counted on top of the detector. Since both the isomers decay by emitting a single gamma, there is no cascade summing. The relative detecior efficiency between the gamma lines at $392 \mathrm{keV}$ and $336 \mathrm{keV}$ was estimated by counting a mixed radionuclide standard containing the nuclides ${ }^{139} \mathrm{Ce}(165.8 \mathrm{keV})$, ${ }^{203} \mathrm{Hg}(279.2 \mathrm{keV}),{ }^{113} \mathrm{Sn}(391.6 \mathrm{keV})$ and ${ }^{85} \mathrm{Sr}(514 \mathrm{keV})$.

\section{Results and discussion}

The ratios of reaction rates per atom $R_{\mathrm{In} 115 \mathrm{~m}} / R_{\mathrm{In} 13 \mathrm{~m}}$ were measured in the three different gamma fields. The results are given in Table 1 . Each one of the values shown in Table 1 is a weighted mean of the results from several trials. The uncertainties quoted consist of uncertainties due to counting statistics and the uncertainties in the determination of the relative efficiency $( \pm 1.7 \%)$. Since both the reactions ${ }^{115} \operatorname{In}\left(\gamma, \gamma^{\prime}\right)^{115 \mathrm{~m}} \operatorname{In}$ and ${ }^{113} \operatorname{In}\left(\gamma, \gamma^{\prime}\right)^{113 \mathrm{~m}} \mathrm{In}$ are induced in the same target, any systematic bias due to positioning the sample during irradiation and counting will cancel out when the ratio of the reaction rates is taken. It is evident from Table 1 that the measured ratios in the three fields are not only very close to each other, but are close to unity as well. The fact that the same value for the ratio was measured in the three gamma fields might lead one to believe either that the excitation functions of both the reactions have the same shape or that the gamma spectra do. For the first few excited levels of the two nuclides, the probability with which they populate the isomeric level was calculated based on the spin parity assignments [10,11]. These are tabulated in Tables 2 and 3. The calculation reveals a striking resemblance between the two cases. The fact that a value close to unity was measured for the reaction rate ratio in each of the three irradiation fields indicates that the number of activation levels and the probabilities with which they populate the isomeric levels must indeed be similar for the nuclides ${ }^{115} \mathrm{In}$ and ${ }^{113} \mathrm{In}$. In otherwords, not only must the cross-section shapes be similar for the two $\left(\gamma, \gamma^{\prime}\right)$ reactions, but the magnitudes of the cross-sections must be almost equal as well. Booth and Brownson [3] have plotted the excitation functions for the two reactions and have concluded that they were almost
Table 2

First few excited levels of ${ }^{115}$ In and the probability with which they populate the isomeric level

\begin{tabular}{cll}
\hline Energy level (keV) & Spin and parity & Probability \\
\hline 0 & $(9 / 2)^{+}$ & - \\
336.24 & $(1 / 2)^{-}$ & - \\
597.14 & $(3 / 2)^{-}$ & 1 \\
828.60 & $(3 / 2)^{+}$ & 1 \\
864.13 & $(1 / 2)^{+}$ & 0.999 \\
933.78 & $(7 / 2)^{+}$ & 0 \\
941.43 & $(5 / 2)^{+}$ & 0.999 \\
1041.50 & $(5 / 2)^{-}$ & 0.910 \\
1078.20 & $(5 / 2)^{+}$ & 0.998 \\
1132.57 & $(11 / 2)^{+}$ & 0 \\
1290.59 & $(13 / 2)^{+}$ & 0 \\
1463.30 & $(7 / 2)^{+}$ & 0.359 \\
\hline
\end{tabular}

identical. This is not surprising, when one examines the level structure of the two indium nuclides. Both of them have an odd proton that occupies the level $g_{9 / 2^{+}},{ }^{115}$ In has two more neutrons than ${ }^{113} \mathrm{In}$, but these are expected to be paired.

The reaction rate ratio $R_{\mathrm{In} 115 \mathrm{~m}} / R_{\mathrm{In} 113 \mathrm{~m}}$ was also measured at low reactor powers, by irradiating indium in the MDRF alongside the south face of the core. This was done to estimate of how different the neutron induced reaction rate ratio was, when compared to the gamma induced ratio. Indium foils, $0.5 \mathrm{in}$. in diameter and $0.020 \mathrm{in}$. thick, were irradiated for $30 \mathrm{~min}$, with the reactor power at $10 \mathrm{~kW}$ and $20 \mathrm{~kW}$. After waiting for a suitable period of time to let the ${ }^{116 \mathrm{~m}}$ In activity decrease sufficiently, the ${ }^{115 \mathrm{~m}}$ In and ${ }^{113 \mathrm{~m}}$ In activities were measured using the high purity $\mathrm{Ge}$ detector. Even at low reactor powers, the ${ }^{115 \mathrm{~m}} \mathrm{In}$ and ${ }^{113 \mathrm{~m}}$ In activities will be predominantly due to the threshold reactions ${ }^{115} \operatorname{In}\left(n, n^{\prime}\right)^{115 m}$ In and ${ }^{113} \operatorname{In}\left(n, n^{\prime}\right)^{113 m}$ In respectively. Intriguingly, the measured reaction rate ratios were

Table 3

First few excited levels of ${ }^{113}$ In and the probability with which they populate the isomeric level

\begin{tabular}{cll}
\hline Energy level (keV) & Spin and parity & Probability \\
\hline 0 & $(9 / 2)^{+}$ & - \\
391.69 & $(1 / 2)^{-}$ & - \\
646.76 & $(3 / 2)^{-}$ & 1 \\
1024.25 & $(5 / 2)^{+}$ & 0.999 \\
1029.71 & $(1 / 2)^{+}$ & 1 \\
1064.16 & $(3 / 2)^{+}$ & 0.999 \\
1106.36 & $(5 / 2)^{-}$ & 0.999 \\
1131.46 & $(5 / 2)^{+}$ & 0.999 \\
1173.10 & $(11 / 2)^{+}$ & 0 \\
1191.07 & $(7 / 2)^{+}$ & 0.018 \\
1344.80 & $(13 / 2)^{+}$ & 0 \\
1509.40 & $(7 / 2)^{+}$ & 0.451 \\
& &
\end{tabular}


Table 4

Measured ratios of $\left(n, n^{\prime}\right)$ reaction rates of Indium in the MDRF

\begin{tabular}{ll}
\hline Reactor power & $R_{\ln 115 \mathrm{~m}} / R_{113 \mathrm{~m}}$ \\
\hline $10 \mathrm{~kW}$ & $1.257 \pm 0.032$ \\
$20 \mathrm{~kW}$ & $1.270 \pm 0.029$ \\
\hline
\end{tabular}

close to the values measured in the gamma fields. The results are given in Table 4 . Since the neutron induced ratios are also close to unity, this indicates that the excitation functions for the two $\left(n, n^{\prime}\right)$ reactions are very similar in shape as well as in magnitude. Smith and Meadows have measured the differential cross-sections of the reactions ${ }^{115} \operatorname{In}\left(\mathrm{n}, \mathrm{n}^{\prime}\right)^{115 \mathrm{~m}}$ In and ${ }^{113} \operatorname{In}\left(\mathrm{n}, \mathrm{n}^{\prime}\right)^{113 \mathrm{~m}}$ In and have found the excitation functions to be almost the same [12]. The ratio of spectrum averaged cross-sections for the two reactions were calculated by folding the reference neutron spectrum in the MDRF with the differential cross-sections. The cross-sections for the ${ }^{115} \operatorname{In}\left(n, n^{\prime}\right)^{115 m}$ In were obtained from the ENDFB-V library. For the reaction ${ }^{113} \mathrm{In}$ $\left(n, n^{\prime}\right)^{113 m}$ In the cross-sections were compiled from the literature $[12,13]$. The calculated ratio of the spectrum averaged cross-sections $\langle\sigma\rangle_{\ln 115\left(n, n^{\prime}\right)} /\langle\sigma\rangle_{\ln 113\left(n, n^{\prime}\right)}$ is 1.20 , which is quite close to the measured reaction rate ratio. This provides further proof to the fact that the excitation functions of the two $\left(n, n^{\prime}\right)$ reactions are very similar in shape and in magnitude.

Since the excited levels of the two nuclides ${ }^{115} \mathrm{In}$ and ${ }^{113}$ In lie in the same energy regime and since the probability with which the excited levels populate the isomeric state are comparable, the measured reaction rate ratio is close to unity. This is true, if one considers the $\left(\gamma, \gamma^{\prime}\right)$ reactions or the $\left(n, n^{\prime}\right)$ reactions in the two indium nuclides.

\section{Conclusions}

The exciation functions for the reactions ${ }^{115} \operatorname{In}(\gamma$, $\left.\gamma^{\prime}\right)^{115 \mathrm{~m}}$ In and ${ }^{113} \operatorname{In}\left(\gamma, \gamma^{\prime}\right)^{113 \mathrm{~m}} \mathrm{In}$ are similar in shape and magnitude. Hence the reaction rate ratio of these two reactions will not yield any information about the gamma spectrum in an irradiation field. Other $\left(\gamma, \gamma^{\prime}\right)$ reaction rate ratios could be used to characterize gamma fields, provided the excitation functions of the two reactions have different shapes. Efforts are underway to measure the reaction rate ratios $R_{\mathrm{In} 115 \mathrm{~m}} / R_{\mathrm{Cd} 11 \mathrm{~m}}$ and $R_{\mathrm{In} 115 \mathrm{~m}} / R_{\mathrm{Sr} 87 \mathrm{~m}}$ in the ${ }^{60}$ Co field.

\section{References}

[1] E. Guth, Phys. Rev. 59 (1941) 325.

[2] B.T. Chertok and E.C. Booth, Nucl. Phys. 66 (1965) 230.

[3] E.C. Booth and J. Brownson, Nucl. Phys. A 98 (1967) 529.

[4] A. Veres, Atom. Energy Rev. 18 (1980) 271.

[5] A. Ljubicic, K. Pisk and B.A. Logan, Phys. Rev. C 23 (1981) 2238.

[6] K. Yoshihara et al., Phys. Rev. C 33 (1986) 728.

[7] M Kremar, A. Ljubicic, B.A. Logan and M. Bistrovic, Nucl. Instr. and Meth. A 255102 (1987) 99.

[8] C.B. Collins et al., Phys. Rev. C 38 (1988) 1852.

[9] A.I. Hawari, R. Venkataraman, R.F. Fleming, C.E. Elsenhauer, E.D. McGarry and J. Grundl, The Materials Dosimetry Reference Facility, Reactor Dosimetry ASTM STP 1228 (1994).

[10] J. Blachot, Nucl. Data Sheets (1990)

[11] J. Blachot and G. Marguier, Nucl. Data Sheets (1992).

[12] D.L. Smith and J.W. Meadows, Nucl. Sci Eng. 60 (1976) 319.

[13] D.C. Santry and J.P Butler, Bull. Am. Phys. Soc. 20 (1975) 171. 